\title{
Far-field super-resolution imaging by nonlinearly excited evanescent waves
}

\author{
Zhihao Zhou $\odot,{ }^{a}{ }^{a}$ Wei Liu, ${ }^{b}$ Jiajing He, ${ }^{a}$ Lei Chen, ${ }^{b}$ Xin Luo, ${ }^{a}$ Dongyi Shen, ${ }^{b}$ Jianjun Cao, ${ }^{c}$ Yaping Dan, ${ }^{a}$ \\ Xianfeng Chen, ${ }^{b}$ and Wenjie Wan $\mathbb{1}^{a, b, *}$ \\ aShanghai Jiao Tong University, University of Michigan-Shanghai Jiao Tong University Joint Institute, State Key Laboratory of Advanced Optical \\ Communication Systems and Networks, Shanghai, China \\ 'Shanghai Jiao Tong University, School of Physics and Astronomy, MOE Key Laboratory for Laser Plasmas and Collaborative Innovation Center of IFSA, \\ Shanghai, China \\ 'Jiangnan University, School of Science, Wuxi, China
}

\begin{abstract}
Abbe's resolution limit, one of the best-known physical limitations, poses a great challenge for any wave system in imaging, wave transport, and dynamics. Originally formulated in linear optics, the Abbe limit can be broken using nonlinear optical interactions. We extend the Abbe theory into a nonlinear regime and experimentally demonstrate a far-field, label-free, and scan-free super-resolution imaging technique based on nonlinear four-wave mixing to retrieve near-field scattered evanescent waves, achieving a sub-wavelength resolution of $\lambda / 5.6$. This method paves the way for numerous new applications in biomedical imaging, semiconductor metrology, and photolithography.
\end{abstract}

Keywords: nonlinear optics; four-wave mixing; evanescent wave; super-resolution; Fourier ptychography.

Received Dec. 23, 2020; revised manuscript received Feb. 25, 2021; accepted for publication Mar. 11, 2021; published online Apr. 1, 2021.

(C) The Authors. Published by SPIE and CLP under a Creative Commons Attribution 4.0 Unported License. Distribution or reproduction of this work in whole or in part requires full attribution of the original publication, including its $\mathrm{DOI}$.

[DOI: 10.1117/1.AP.3.2.025001]

\section{Introduction}

The spatial resolution of an imaging system is limited by the Abbe theory, ${ }^{1}$ posing a great challenge for many areas such as biomedical imaging, astronomy, and photolithography. For example, direct live images are crucial to understanding biological processes at the subcellular level, e.g., virus behaviors; ${ }^{2,3}$ nanofabrication by photolithography on semiconductor chips is also limited by diffraction. According to Abbe's theory, subwavelength image features are usually associated with near-field evanescent waves, which decay exponentially with distance similar to electron wavefunctions in quantum tunneling. ${ }^{4}$ Insufficient far-field detection of these evanescent fields in conventional optics with finite numerical apertures (NAs) ultimately precludes imaging resolution better than $\lambda / 2$. Like scanning tunneling electron techniques in condensed-matter physics converting tunneling currents into conducting ones, ${ }^{5}$ a near-field scanning optical microscope (NSOM) ${ }^{6}$ can improve the resolution by converting evanescent waves into propagating ones but requires near-field, nanometer-scale scanning. Similarly,

*Address all correspondence to Wenjie Wan, wenjie.wan@sjtu.edu.cn configurations involving evanescent-wave illuminations such as total internal reflection (TIR), ${ }^{7}$ waveguide, ${ }^{8,9}$ and surface plasmon polaritons $(\mathrm{SPPs})^{10}$ also permit enhanced imaging resolution. Recent advancements in metamaterials manipulate near-field evanescent waves using superlens ${ }^{11}$ and hyperlens, ${ }^{12,13}$ achieving super-resolution imaging but demanding nanofabrication. On the other hand, $x$-ray and electron microscopes, even with nanometer resolution, may be potentially harmful in biomedical applications, and it is still highly desirable to break the Abbe limit and realize super-resolution imaging for optical waves.

Nonlinear optics may offer an alternative way to beat the Abbe limit, which is originally formulated for linear waves. ${ }^{14-16}$ By introducing nonlinear spatial wave mixing into an imaging system, it becomes possible to retrieve those undetected waves in the far field and reconstruct them to improve the resolution, ${ }^{15}$ effectively generalizing the Abbe theory into a nonlinear optics regime. However, evanescent waves are absent in prior works; the imaging resolutions have not reached the wavelength scale yet until this work. Meanwhile, evanescent waves can be manipulated through nonlinear wave mixings ${ }^{16-18}$ based on surface phase-matching conditions, e.g., free-space 
coupling of SPP ${ }^{19,20}$ and dark-field imaging. ${ }^{21}$ These works show a unique way to nonlinearly couple non-propagating evanescent waves with propagating ones into the far field, addressing the key issue for the aforementioned super-resolution imaging problem in the framework of nonlinear Abbe theory. Moreover, it may enable a fluorophore-label-free imaging method in contrast to the existing techniques such as stimulated emission depletion microscopy ${ }^{22}$ stochastic optical reconstruction microscopy, ${ }^{3}$ structured illumination microscopy (SIM) ${ }^{23,24}$ where label-free imaging techniques are highly desirable not only in biomedical imaging ${ }^{25}$ but also in other areas such as semiconductor metrology processes.

In this work, we experimentally show nonlinear wave mixings, including evanescent waves in the framework of nonlinear Abbe theory, and demonstrate a far-field, label-free, and scan-free super-resolution imaging scheme to resolve subwavelength structures on a semiconductor silicon-on-insulator (SOI) wafer. To break the Abbe resolution limit, a nonlinear four-wave mixing (FWM) technique is implemented to excite localized near-field evanescent-wave illumination with large spatial wave vectors such that near-field waves containing the finest sub-wavelength image features can be converted into propagating ones for far-field detection, effectively enlarging the NAs. Combined with an iterative Fourier ptychography (FP) method, ${ }^{26,27}$ the reconstructed images can reach a resolution limit down to $\lambda / 5.6$ with respect to the input probe's wavelength. Moreover, this FWM imaging scheme can also cooperate with nano-slit grating structures, which theoretically can provide additional resolution enhancement. This technique may offer a new way for critical imaging tasks sensitive to fluorescent labels such as biomedical applications, semiconductor processes, and photolithography.

\section{Principles and Methods}

According to Abbe's theory, a target with sub-wavelength structures illuminated by a coherent wave with wave vector $\mathbf{k}$ will cause scattering waves to radiate over a wide-angle into the far field. Meanwhile, a portion of scattered light with large wave vectors becomes evanescent and confined only to the target's surface in the near field. Such evanescent components correspond to portions of large wave-vector magnitude in Fourier space, carrying the finest feature information of the target. How to retrieve these near-field evanescent waves in the far field is the key to beating the Abbe diffraction limit. ${ }^{28}$

Many prior attempts in the linear case have been implemented to tackle this problem. For example, NSOM, ${ }^{6}$ a nano-aperture with extreme confinement of light, can excite localized evanescent waves to illuminate sub-wavelength features. Consequently, the evanescent waves will be scattered from the imaging target and converted into propagating ones carrying sub-wavelength information for far-field detection; however, much demanding near-field scanning is always required. Other approaches such as using TIR, ${ }^{7}$ waveguides ${ }^{8,9}$ and $\mathrm{SPPs}^{10}$ for evanescent-wave illumination have to deeply rely on specific material permittivity; as a result, their maximum resolution is limited. Moreover, fluorescent labeling is usually accompanied in these techniques to help distinguish the signal from background for a better-contrasted image.

On the other hand, recent work of nonlinear wave mixing at the interfaces allows nonlinear wavelength conversion under partial-phase-matching conditions between propagating waves and near-field evanescent waves, ${ }^{19,20,29}$ paving the way for this proposed super-resolution imaging purpose. In this configuration [Fig. 1(a)], a near-field evanescent wave with wave vector $\mathbf{k}_{3, \text { eva }}$ at frequency $\omega_{3}$ can be excited through nonlinear FWM by the far-field pump $\omega_{1}$ and probe $\omega_{2}$. The evanescent wave will be scattered off by the sub-wavelength target into radiating ones at various angles into the far field. With an appropriate imaging reconstruction, the sub-wavelength information can be retrieved from these converted evanescent waves for super-resolution imaging. This technique does not require fluorescent labeling, near-field scanning, or fabricated structures, which are highly demanded in the linear case.

In the spatial-frequency domain, i.e., $k$-space [Fig. 1(b)], this scattering process can be described by in-plane momentum conservation: ${ }^{30}$

$\left|k_{3, x}\right|=\left|k_{3, \mathrm{eva}}-2 \pi / \Lambda\right|<n_{3} k_{3,0}$,

where $k_{3,0}$ is the free-space wavenumber of FWM and $\Lambda$ represents various spatial features of the target. In reality, a generic imaging target contains a wide spectrum of spatial modes, extending to a broad region in $k$-space. Under a normally incident illumination, these spatial modes collected in the far field by an imaging lens with a limited NA lie within the range of $|2 \pi / \Lambda|<$ $\mathrm{NA} \times k_{3,0}$ in $k$-space [Fig. 1(b)]. In contrast, if illuminated by the nonlinearly excited evanescent wave, the effective passband in $k$-space of the same imaging system will be shifted by $\mathbf{k}_{3 \text {,eva }}$ [Fig. 1(b)], meaning that most of the passband lies within the evanescent field of the target's spectrum, which stores the sub-wavelength information. In this manner, these evanescent waves with large wave vectors carrying sub-wavelength details can be retrieved in the far field with a conventional objective lens. If 2D wave-vector-space illumination is adopted, the final accessible region in Fourier space can be extended to the utmost dashed circle [Fig. 1(b)]. Combined with certain reconstruction methods, evanescent fields with different orientations in Fourier space can be replenished like SIM does ${ }^{24}$ which effectively enlarges the NA to include these components and far-field superresolution imaging can be realized.

The key mechanism to excite near-field evanescent waves through FWM can be understood from nonlinear phase-matching conditions at the interface. In linear optics, the laws of reflection and refraction at an interface can be directly deduced from electromagnetic boundary conditions laid out by Fresnel formalism. ${ }^{31}$ Under the same framework, the well-known linear Snell's law can be generalized into a nonlinear regime where light waves are incident onto a nonlinear medium boundary by satisfying boundary conditions for all individual frequencies during nonlinear conversions. ${ }^{32}$ For the case of FWMs, the resulting equations governing reflection and refraction have been developed in Refs. 17 and 29 as well as our prior work, ${ }^{16}$ where the nonlinear reflection law reads

$k_{3} \sin \left(\theta_{3}\right)=2 k_{1} \sin \left(\theta_{1}\right)-k_{2} \sin \left(\theta_{2}\right)$,

where $k_{i}=n_{i} \omega_{i} / c(i=1,2,3)$ are wavenumbers of the pump, probe, and FWM signal beams, respectively; $n_{i}$ are refractive indices of the ambient medium; $\theta_{i}$ represent their incident/ output angles. Effectively, the relations of these transverse wave vectors represent in-plane momentum conservation laws as shown in Fig. 2(a). Such a partial-phase-matching condition only manifests itself near surfaces ${ }^{21,33}$ and can be enhanced by thin-film structures. ${ }^{29}$ More importantly, this reduced-dimension 


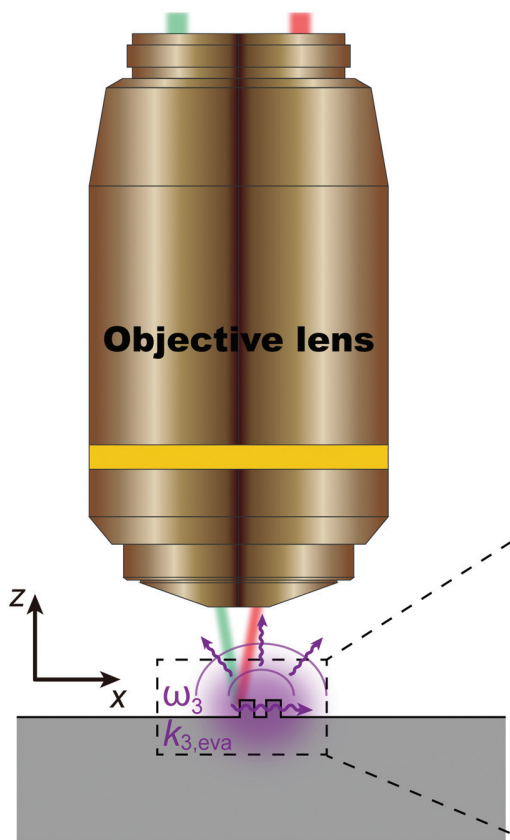

(a)
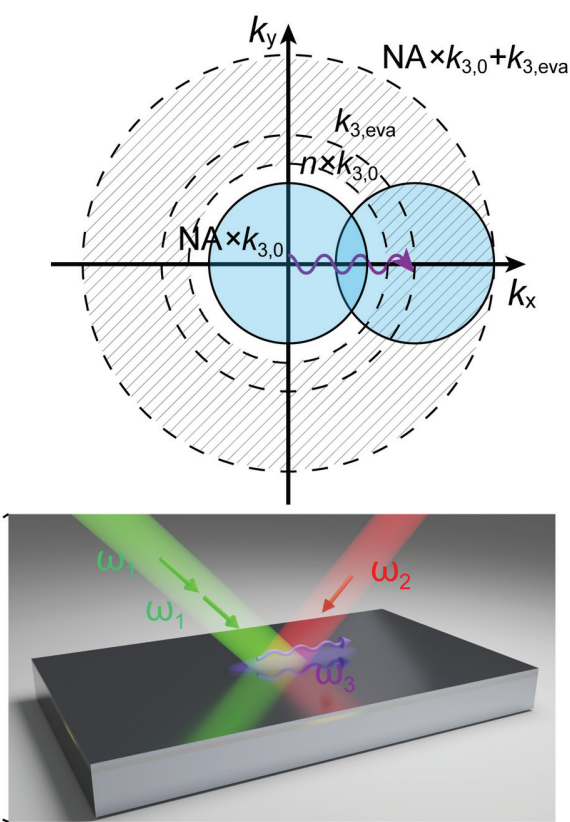

(b)

Fig. 1 The working principle of far-field super-resolution imaging based on nonlinearly excited evanescent waves. (a) Schematic of the far-field super-resolution imaging process with localized evanescent-wave illumination excited by the FWM process at the interface. The zoomed inset shows that the FWM process takes place on nonlinear medium's interface. (b) Mechanism illustrated in Fourier space. NA and $k_{3,0}$ represent numerical aperture and the free-space wavenumber of the FWM signal beam, respectively. $\mathrm{NA} \times k_{3,0}$ determines the cutoff frequency of the imaging system; correspondingly, the blue-circled area represents the system's passband. Stripedshadow regions represent evanescent fields, which carry sub-wavelength details of the imaging target. In the current experiment, we introduce evanescent wave vector $\mathbf{k}_{3 \text {,eva }}$ along $k_{x}$ as illumination light, which can map evanescent fields into propagating ones and extend the accessible region of the target's spectrum on dimension $k_{x}$.

requirement of phase matching can lead to intriguing results where the transverse component of the FWM wave $k_{3, x}$ (denoted by $k_{3, \text { eva }}$ for evanescent waves later) can exceed its free-space magnitude $k_{3}$, i.e., propagating waves. As a consequence, its corresponding vertical component becomes purely imaginary according to $k_{3, z}=i \sqrt{k_{3, x}^{2}-k_{3}^{2}}$, making the wave evanescent. Ideally, the maximum wavenumber of evanescent-wave illumination can be as large as $2 k_{1}+k_{2}$ (if the pump/probe beams' incident angles are $\sim 90 \mathrm{deg}$ ). The nonlinearly excited evanescent waves are bound at the interface, but with large in-plane wave vectors, which is crucial for the aforementioned scattering imaging process.

Experimentally, we first verify Eq. (2) in a reflection configuration by synchronously launching a pump and a probe beam onto a flat SOI wafer to excite near-field FWM as shown in Fig. 2(a). Here, two pump photons $\lambda_{1}=532 \mathrm{~nm}$ from a frequency-doubled fiber femtosecond laser and a probe photon $\lambda_{2}=780 \mathrm{~nm}$ from a customized optical parametric oscillator interact together, exciting one FWM signal photon $\lambda_{3}=$ $403 \mathrm{~nm}$ (details in the Supplementary Material and Refs. 34 and 35). Both the incident and output beams are collected at the back focal plane [Figs. 2(b) and 2(c)], which vividly reveals the wave vector distributions in Fourier space. The measured reflection angles of FWM waves $\theta_{3}$ exhibit an upward trend with increasing incident angles of the probe $\theta_{2}$ for a given angle $\theta_{1}$ [Fig. 2(d)], well fitted to the theoretical curves predicted by Eq. (2). Furthermore, this reflection angle $\theta_{3}$ can exceed $90 \mathrm{deg}$, making the FWM evanescent. Specifically, for the incident angle $\theta_{1}=30.7 \mathrm{deg}$ (blue curve), the FWM waves become evanescent above the angle $\theta_{2}=25.9 \mathrm{deg}$ (the maximum collected angle of FWM here is limited to 58.9 deg due to the NA) denoted by a gray region in Fig. 2(d), which is the region of interest to explore our evanescent wave-based sub-wavelength imaging. Also, such nonlinearly excited evanescent waves have been previously demonstrated for surface plasmon coupling ${ }^{19,20}$ and dark-field imaging. ${ }^{21}$

To illustrate the case of evanescent wave excitation, we perform a numerical simulation of surface FWM by the finitedifference time-domain method. The calculated $|\mathbf{E}|^{2}$ distribution of the FWM signal shows that the signal wave is localized near the focal spot inside the top silicon layer of the SOI wafer [Fig. 2(e)]. This is a sharp contrast to the nonlinearly excited surface plasmon mode near a metal-dielectric interface, ${ }^{20,36}$ where surface plasmons can further propagate under certain phase-matching conditions. Such nonlinearly excited FWM waves serve as localized evanescent light sources for sub-wavelength imaging purposes later. According to the aforementioned discussions on imaging resolution, it is essential to obtain large transverse wave vectors for excited evanescent waves. As shown 


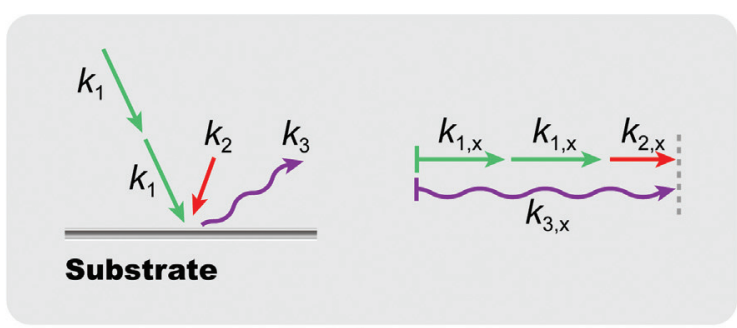

(a)

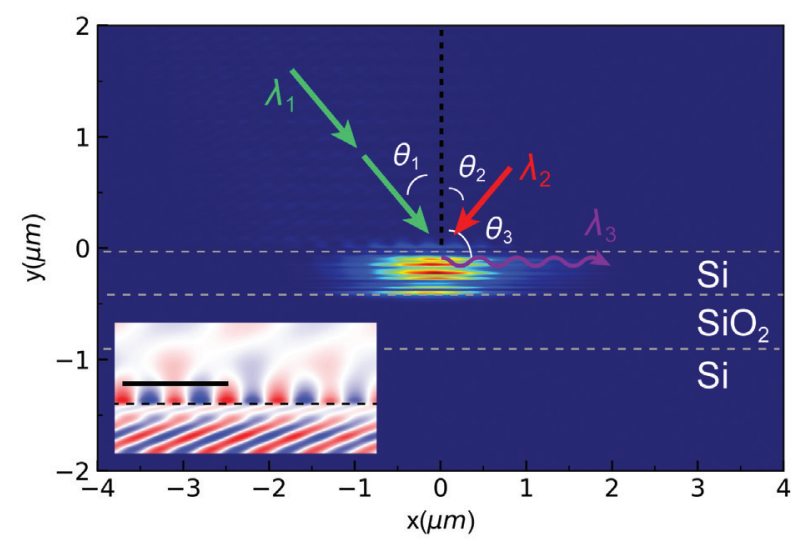

(e)

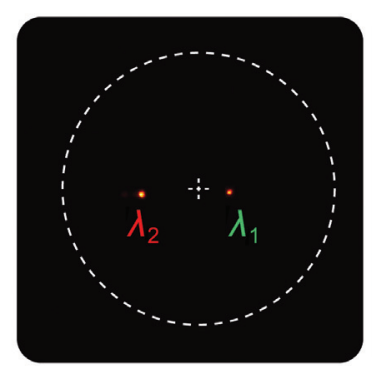

(b)

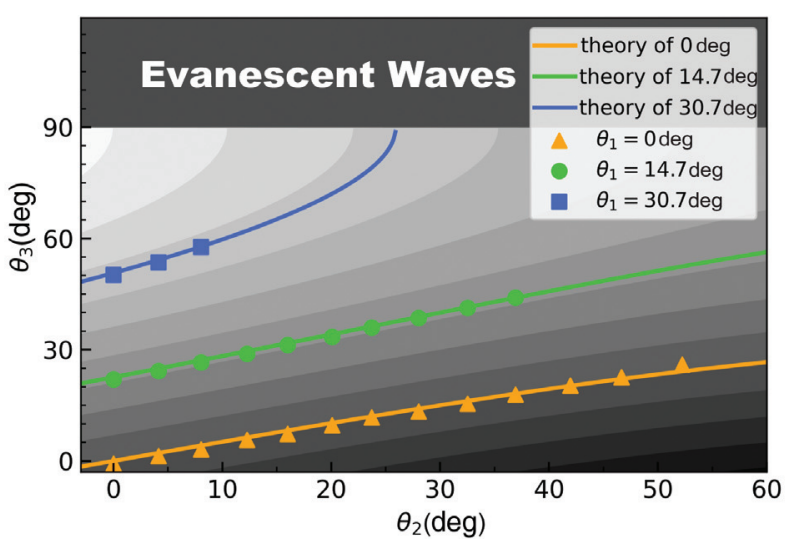

(d)

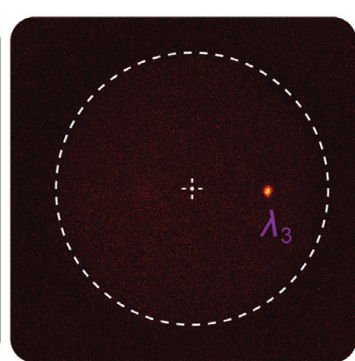

(c)

Fig. 2 Demonstration of wave vector control in surface FWM and local excitation of evanescent waves. (a) Illustration of the FWM process takes place at the interface and the partial-phasematching condition. (b), (c) Fourier space images of reflected pump/probe beams $\lambda_{1}, \lambda_{2}$, and signal beam $\lambda_{3}$ taken by EMCCD, characterizing incident angles, and output angle, respectively. (d) Dependence of FWM output angle $\theta_{3}$ on probe incident angle $\theta_{2}$ under different pump angles $\theta_{1}$. (e) Numerical simulation result of FWM signal field distribution, which is the case of an evanescent wave with a large transverse wave vector $\mathbf{k}_{3, \text { eva }}$ localized at the top film of SOI. The inset shows the signal's amplitude variation along with the interface, where the scale bar represents the wavelength of FWM.

in the inset of Fig. 2(e), the effective wavelength of such an evanescent wave has been reduced to $\sim 200 \mathrm{~nm}$, half of its free-space wavelength $(\sim 403 \mathrm{~nm})$, leading to the relation of wave vectors as $k_{3 \text {,eva }}=2 k_{3,0}$, which is the same result according to Eq. (2) for given input angles in the simulation $\left(\theta_{1}, \theta_{2}=40 \mathrm{deg}\right)$. Effectively, by varying the input angles of the pump and probe beams, we manage to locally excite evanescent waves with variable wave vectors. Such an FWM process enables an active, flexible manner to control the signal beam's wave vector for the imaging process later.

\section{Results}

To demonstrate evanescent wave-based sub-wavelength imaging, we compare image qualities under several illumination schemes in Fig. 3. Consider a double-slit with width $w$ and center-to-center distance $a$ [Fig. 3(j)] illuminated by an evanescent wave with wave vector $\mathbf{k}_{3 \text {,eva }}$, the reflected far-field image through an NA-limited imaging system in the spatial domain can be formulated $\mathrm{as}^{8}$

$I(x)=\left|s(x)+\exp \left(i k_{3, \mathrm{eva}} a\right) s(x-a)\right|^{2}$,

where $s(x)=h(x) *\left[\exp \left(i k_{3, \text { eva }} x\right) \times \operatorname{rect}\left(\frac{x}{w}\right)\right]$ is the amplitude distribution of a single slit function centered at $x=0$, i.e., $\operatorname{rect}\left(\frac{x}{w}\right)$, and $h(x)$ is point spread function of the imaging system. The extra phase term induced by the illumination beam $\exp \left(i k_{3, \mathrm{eva}} x\right)$ effectively shifts the angular spectrum of the images in $k$-space by $k_{3, \text { eva }}{ }^{37}$ as shown in Fig. 3(b), which represents the finer portion of the image with better resolution. For example, the relative phase term $\exp \left(i k_{3, \text { eva }} a\right)$ between the first $s(x)$ and the second slit $s(x-a)$ can be out-of-phase in the regime of $\frac{\pi}{2}<k_{3, \text { eva }} a<\frac{3 \pi}{2}$, forming a dip in the far-field image [Figs. 3(e) and 3(h)]. In contrast, the same feature cannot be resolved under normal illumination when $k_{2, x}=0$ [Figs. 3(d) and $3(\mathrm{~g})]$.

Similar ideas have been realized by TIR, ${ }^{7}$ SPPs,${ }^{10}$ and guided waves $^{8,9}$ to achieve super-resolution along a single direction in $k$-space. For a complex target, coherent artifacts will occur and the key issue is to acquire multiple angle-resolved super-resolution images and combine them together to restore a complete super-resolution image. Here, we reconstruct sub-wavelength images using a computational imaging technique named $\mathrm{FP}^{26,27}$ by stacking multiple low-resolution images with pre-determined illumination wave vectors and iteratively reconstructing a final image with much-enhanced resolution as well as eliminated coherent defects (see Supplementary Material). Previously, this technique has shown great successes in wide-field, highresolution imaging ${ }^{26}$ and fast live-cell imaging. ${ }^{27}$ In our case, 


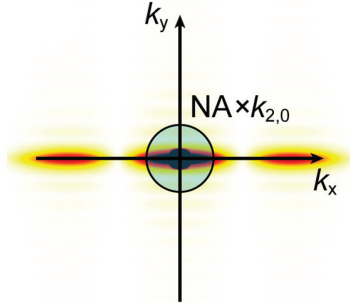

(a)

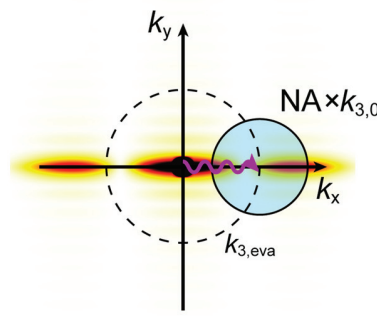

(b)

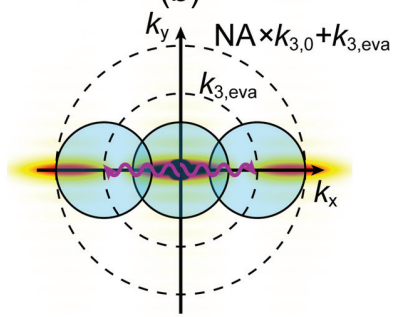

(c)

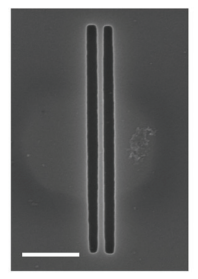

(j)
Simulation

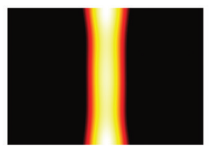

(d)

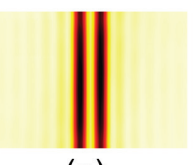

(e)

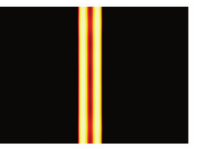

(f)

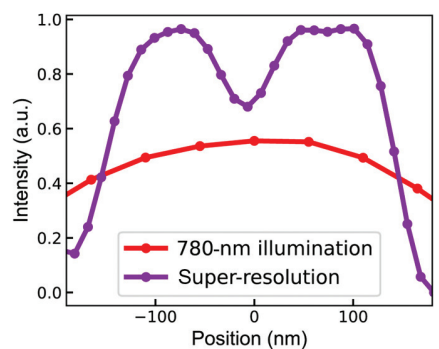

(k)

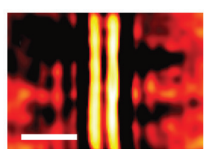

(i)

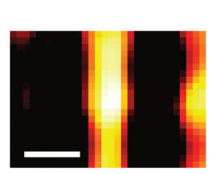

(g)

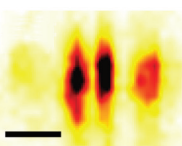

(h)

Fig. 3 Demonstration of super-resolution imaging using FWM evanescent-wave illumination. Fourier space representation of (a) the probe beam at $780 \mathrm{~nm}$ with normal illumination, (b) FWM signal at $403 \mathrm{~nm}$ with $\mathbf{k}_{3 \text {,eva }}$ illumination, and (c) the complete imaging method (d)-(f). Simulated images of a pair of 90-nm-wide slits spaced $50 \mathrm{~nm}$ apart, corresponding to cases of (a)-(c). (g)-(i) Experimental results. Scale bar: $500 \mathrm{~nm}$. (j) Scanning electron microscopy image of the two-slit target. (k) Inverse-intensity cross-section comparison of probe beam normal illumination image and proposed super-resolution method.

we extend this technique into a nonlinear optics regime by including nonlinearly excited evanescent illumination, due to flexible control over $\mathbf{k}_{3}$. Using such an FP technique, a complete image of the double slits can be reconstructed by stacking three portions in $k$-space along the $k_{x}$ axis [Fig. 3(c)], which includes two evanescent waves at the frequency $\omega_{3}$ with opposite directions $\left(k_{3, \text { eva1 }}=1.56 k_{3,0}, k_{3, \text { eva2 }}=-1.58 k_{3,0}\right)$ and one nearnormal illumination $\left(k_{3, \text { eva } 3}=-0.12 k_{3,0}\right)$. After the iteration converges, an intensity dip appears in the reconstructed image, making the two slits resolvable now [Fig. 3(i)]. Experimentally, a pair of 90-nm-width slits spaced $50 \mathrm{~nm}$ apart on SOI substrate [Fig. 3(j)] can be successfully resolved using this technique as

shown in Figs. 3(i) and 3(k). The image resolution is sharply improved, in contrast, over those images formed under probe beam normal illumination [Fig. 3(g)] and solely signal illumination at $k_{3, \text { eval }}=1.56 k_{3,0}[$ Fig. $3(\mathrm{~h})]$. These results are also well confirmed by the numerical simulation [Figs. 3(d) and 3(e)]. Due to the current image system's limitation, e.g., NA, the estimated image resolution is around $140 \mathrm{~nm}$ (center-tocenter distance). To further shrink the gap distance and improve the system resolution, the wavenumber $k_{3 \text {,eva }}$ must be increased accordingly.

For demonstration of super-resolution over a larger field-ofview, we implement this sub-wavelength imaging technique for a nano-slit array with $110-\mathrm{nm}$ slit width and 400-nm period [Figs. 4(a)-4(d)]. The fine features are clearly revealed using this technique [Figs. 4(d) and 4(e)] as compared to the blurred one under the probe normal illumination [Fig. 4(c)]. Image contrast has also been significantly improved as shown in Fig. 4(e) because such an FP technique helps to replenish the high spatial frequency parts of images that majorly contribute to a high signal-to-noise ratio (SNR). Meanwhile, localized near-field evanescent waves serve as excellent dark-field illumination sources. After filtering out the pump/probe frequencies, they clear out background noises for the high SNR. In addition, this iterative reconstruction algorithm enables phase-retrieval ability in revealing sensitive information such as depth/height ${ }^{38}$ and has potential for topographic imaging applications in the future.

Interestingly, deposited grating structures can also provide a platform facilitating evanescent wave excitation. The grating introduces an additional in-plane momentum $|\mathbf{G}|=| \pm 2 \pi / D|$ (first-order), which is inversely proportional to the spatial period $D$, to the FWM process. These grating modes can be involved in the nonlinear process ${ }^{14,15}$ and affect the excited signal beam's wave vector. ${ }^{29}$ Under this new scheme, the modified phasematching condition should include the grating momentum term as

$k_{3} \sin \left(\theta_{3}\right)=2 k_{1} \sin \left(\theta_{1}\right)-k_{2} \sin \left(\theta_{2}\right) \pm n G$.

Accordingly, Fig. 4(f) exhibits the angle dependence of FWM on a grating with a period of $320 \mathrm{~nm}$ extracted from Fourier space. For a fixed input angle $\theta_{2}=41 \mathrm{deg}$, the measured FWM angles are well-matched with theoretical results from Eq. (4) for both \pm 1 st and -2 nd grating orders. It is noteworthy that +1 st grating mode tends to allow larger FWM output angles than the other two orders even for small input $\theta_{1}$, showing potential for promoting the FWM wavenumber. This can also be understood from in-plane momentum conservation laws [Figs. 4(g) and 4(h)], where the additional grating momentum of +1 st order further enlarges the FWM wave vector into the evanescent regime. For instance, given the pump/probe incident angles at their NA's maximum, the maximum wave vector of the FWM signal can be as large as $4.6 k_{3,0}$. This implies that the image resolution can be further enhanced if the imaging target is placed on top of this grating, which requires future investigation.

\section{Discussions}

In the current configuration, the resolution of our imaging technique can reach around $140 \mathrm{~nm}, \sim \lambda / 5.6$ with respect to the probe's wavelength. However, it can further be improved if the maximum evanescent wave vector $2 k_{1}+k_{2}$ is utilized, which requires that the pump/probe beams are illuminated perpendicularly 
Simulation

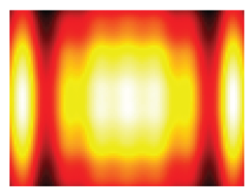

(a)

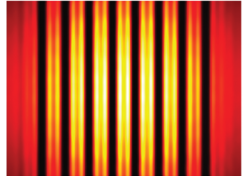

(b)
Experiment

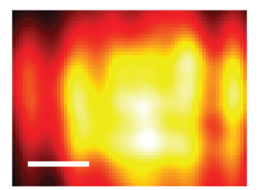

(c)

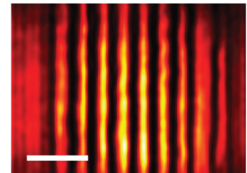

(d)
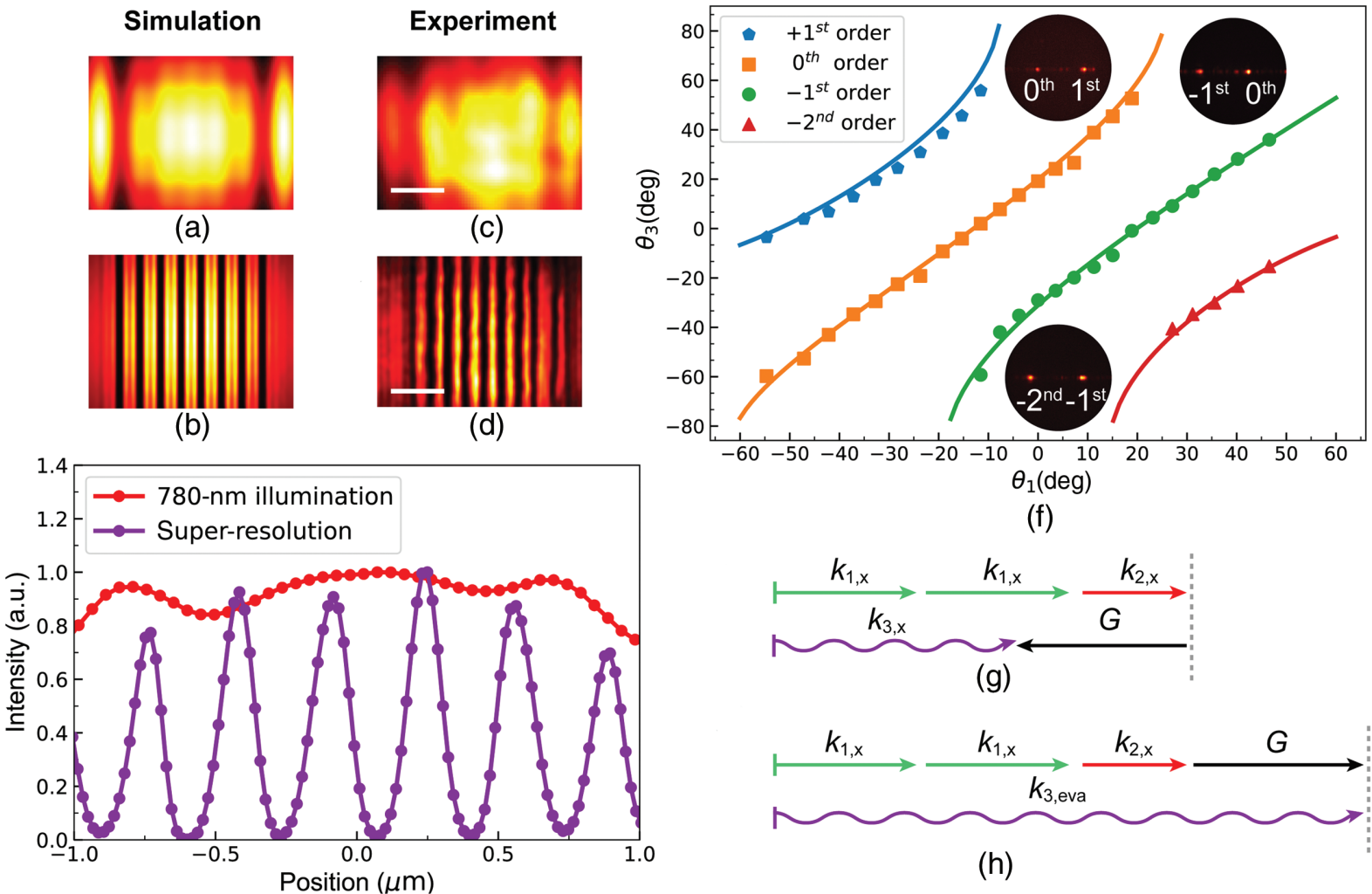

(f)

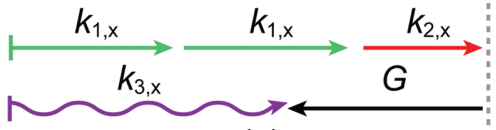

(g)

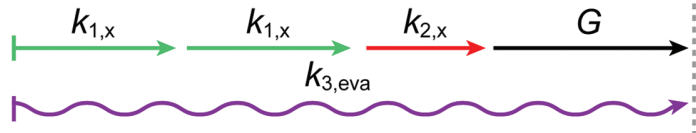

(h)

(e)

Fig. 4 Super-resolved nano-slit grating and evanescent wave excitation on the grating. (a) and (b) Simulated images of a slit array with 110-nm slit width and 400-nm period by probe beam illumination and our super-resolution method, respectively. (c) and (d) Experimental results. Scale bar: $1 \mu \mathrm{m}$. (e) Cross-section comparison of the two cases, showing a great improvement in resolution by our method. (f) Experimental demonstration of the modified partial-phase-matching condition mixed with grating modes when $\theta_{2}=41 \mathrm{deg}$, solid lines are the theoretical prediction calculated from Eq. (4), and insets show typical $k$-space images used to estimate the signal's output angles. (g) Partial-phase-matching condition mixed with grating modes $-G$, making the FWM signal become propagating waves. (h) Partial-phase-matching condition mixed with $G$, resulting in a further increment of $k_{3 \text {,eva }}$.

to the direction of observation, similar to light-sheet microscopy. ${ }^{39}$ In the future, a UV laser source with wavelengths smaller than $300 \mathrm{~nm}$ and the assistance from the aforementioned nanograting structures may finally put the resolution limit down to a few tens of nanometers, showing great potential in subwavelength imaging. We expect that this technique could be especially beneficial for semiconductor metrology, where UV absorption would not be an issue for semiconductor materials. As for biomedical imaging applications, our technique offers a label-free, scan-free, and far-field super-resolution capability, which is much demanded in this area. To avoid possible laser damage to the bioimaging samples, we may consider previous similar approaches using nonlinearly excited SPPs to separate laser focus and light-sensitive biosamples. ${ }^{36}$ But compared with fixed wavelength SPPs/LSPs illumination, our method allows continuous varying of wave vectors, which enables nonlinear FP reconstruction to produce better imaging qualities. Moreover, given the nonlinear nature of FWM, we also expect our method combined with the coherent anti-Stokes Raman scattering technique ${ }^{25,40,41}$ together may offer chemical-specified, far-field super-resolution imaging by pairing pump/probe beams' frequencies according to molecules' vibrational energy. Last, in a reversed manner, such nonlinearly excited evanescent waves with large spatial wave vectors are capable of focusing light into tiny spots below the diffraction limit by constructively interfering with each other, showing promise for a new type of highresolution photolithography mechanism. ${ }^{42}$

\section{Conclusions}

We experimentally demonstrate a super-resolution imaging method based on surface nonlinear FWM-excited evanescentwave illumination, which enables label-free, far-field imaging well beyond Abbe's diffraction limit. Such locally excited evanescent waves may also be beneficial for other applications beyond imaging, such as photolithography.

\section{Acknowledgments}

This work was supported by the National Key Research and Development Program (Grant Nos. 2016YFA0302500 and 2017YFA0303700); National Natural Science Foundation of China (Grant Nos. 92050113 and 11674228); Shanghai MEC Scientific Innovation Program (Grant No. E00075). 


\section{References}

1. E. Abbe, "Beiträge zur Theorie des Mikroskops und der mikroskopischen Wahrnehmung," Arch. für mikroskopische Anat. 9(1), 413-468 (1873).

2. E. Betzig and J. K. Trautman, "Near-field optics: microscopy, spectroscopy, and surface modification beyond the diffraction limit," Science 257(5067), 189-195 (1992).

3. M. J. Rust, M. Bates, and X. Zhuang, "Sub-diffraction-limit imaging by stochastic optical reconstruction microscopy (STORM)," Nat. Methods 3(10), 793-795 (2006).

4. D. J. Griffiths and D. F. Schroeter, Introduction to Quantum Mechanics, Cambridge University Press (2018).

5. G. Binnig et al., "Tunneling through a controllable vacuum gap," Appl. Phys. Lett. 40(2), 178-180 (1982).

6. E. A. Ash and G. Nicholls, "Super-resolution aperture scanning microscope," Nature 237(5357), 510-512 (1972).

7. P. von Olshausen and A. Rohrbach, "Coherent total internal reflection dark-field microscopy: label-free imaging beyond the diffraction limit," Opt. Lett. 38(20), 4066-4069 (2013).

8. X. Liu et al., "Fluorescent nanowire ring illumination for widefield far-field subdiffraction imaging," Phys. Rev. Lett. 118(7), 076101 (2017).

9. A. Archetti et al., "Waveguide-PAINT offers an open platform for large field-of-view super-resolution imaging," Nat. Commun. 10(1), 1267 (2019).

10. F. Wei et al., "Wide field super-resolution surface imaging through plasmonic structured illumination microscopy," Nano Lett. 14(8), 4634-4639 (2014).

11. N. Fang et al., "Sub-diffraction-limited optical imaging with a silver superlens," Science 308(5721), 534-537 (2005).

12. Z. Jacob, L. V. Alekseyev, and E. Narimanov, "Optical hyperlens: far-field imaging beyond the diffraction limit," Opt. Express 14(18), 8247-8256 (2006).

13. Z. Liu et al., "Far-field optical hyperlens magnifying sub-diffraction-limited objects," Science 315(5819), 1686 (2007).

14. C. Barsi, W. Wan, and J. W. Fleischer, "Imaging through nonlinear media using digital holography," Nat. Photonics 3(4), 211-213 (2009).

15. C. Barsi and J. W. Fleischer, "Nonlinear Abbe theory," Nat. Photonics 7(8), 639-643 (2013).

16. J. Cao et al., "Metal-free flat lens using negative refraction by nonlinear four-wave mixing," Phys. Rev. Lett. 113(21), 217401 (2014).

17. S. Palomba et al., "Optical negative refraction by four-wave mixing in thin metallic nanostructures," Nat. Mater. 11(1), 34-38 (2012).

18. J. Cao et al., "Nonlinear negative refraction by difference frequency generation," Appl. Phys. Lett. 108(19), 191101 (2016).

19. S. Palomba and L. Novotny, "Nonlinear excitation of surface plasmon polaritons by four-wave mixing," Phys. Rev. Lett. 101(5), 056802 (2008).

20. J. Renger et al., "Free-space excitation of propagating surface plasmon polaritons by nonlinear four-wave mixing," Phys. Rev. Lett. 103(26), 266802 (2009).

21. H. Harutyunyan et al., "Nonlinear dark-field microscopy," Nano Lett. 10(12), 5076-5079 (2010).

22. S. W. Hell and J. Wichmann, "Breaking the diffraction resolution limit by stimulated emission: stimulated-emission-depletion fluorescence microscopy," Opt. Lett. 19(11), 780-782 (1994).

23. M. G. L. Gustafsson, "Surpassing the lateral resolution limit by a factor of two using structured illumination microscopy," J. Microsc. 198(2), 82-87 (2000).

24. M. G. L. Gustafsson, "Nonlinear structured-illumination microscopy: wide-field fluorescence imaging with theoretically un- limited resolution," Proc. Natl. Acad. Sci. U. S. A. 102(37), 13081-13086 (2005)

25. C. W. Freudiger et al., "Label-free biomedical imaging with high sensitivity by stimulated Raman scattering microscopy," Science 322(5909), 1857-1861 (2008).

26. G. Zheng, R. Horstmeyer, and C. Yang, "Wide-field, high-resolution Fourier ptychographic microscopy," Nat. Photonics 7(9), 739-745 (2013).

27. L. Tian et al., "Multiplexed coded illumination for Fourier ptychography with an LED array microscope," Biomed. Opt. Express 5(7), 2376-2389 (2014).

28. J. B. Pendry, "Negative refraction makes a perfect lens," Phys. Rev. Lett. 85(18), 3966-3969 (2000).

29. J. Renger et al., "Surface-enhanced nonlinear four-wave mixing," Phys. Rev. Lett. 104(4), 046803 (2010).

30. L. Novotny and B. Hecht, Principles of Nano-Optics, Cambridge University Press (2012).

31. M. Born and E. Wolf, Principles of Optics: Electromagnetic Theory of Propagation, Interference and Diffraction of Light, Elsevier (2013).

32. N. Bloembergen and P. S. Pershan, "Light waves at the boundary of nonlinear media," Phys. Rev. 128(2), 606-622 (1962).

33. X. Guo et al., "Nonreciprocal metasurface with space-time phase modulation," Light Sci. Appl. 8(1), 123 (2019).

34. J. Cao et al., "Femtosecond OPO based on MgO:PPLN synchronously pumped by a $532 \mathrm{~nm}$ fiber laser," Laser Phys. 27(5), 055402 (2017).

35. R. W. Boyd, Nonlinear Optics, Academic Press (2008).

36. B. Simkhovich and G. Bartal, "Plasmon-enhanced four-wave mixing for superresolution applications," Phys. Rev. Lett. 112(5), 056802 (2014).

37. J. W. Goodman, Introduction to Fourier Optics, Roberts and Company Publishers (2005).

38. Q. Liu et al., "Surface wave illumination Fourier ptychographic microscopy," Opt. Lett. 41(22), 5373-5376 (2016).

39. H. U. Dodt et al., "Ultramicroscopy: three-dimensional visualization of neuronal networks in the whole mouse brain," Nat. Methods 4(4), 331-336 (2007).

40. A. Zumbusch, G. R. Holtom, and X. S. Xie, "Three-dimensional vibrational imaging by coherent anti-Stokes Raman scattering," Phys. Rev. Lett. 82(20), 4142-4145 (1999).

41. L. Gong et al., "Higher-order coherent anti-Stokes Raman scattering microscopy realizes label-free super-resolution vibrational imaging," Nat. Photonics 14(2), 115-122 (2020).

42. X. Luo and T. Ishihara, "Subwavelength photolithography based on surface-plasmon polariton resonance," Opt. Express 12(14), 3055-3065 (2004).

Zhihao Zhou is currently a PhD student at Shanghai Jiao Tong University. He received his BS degree from the Department of Physics, East China Normal University in 2017. His current research interests include nonlinear optics and nanophotonics.

Wenjie Wan received his BE degree from the Hong Kong University of Science and Technology and his PhD from Princeton University in 2010. After one-year postdoctoral training at Yale University, he joined the faculty of Shanghai Jiao Tong University in 2011, where he is currently an associate professor. His current research interests include nonlinear optics, nanophotonics, and optical microcavity.

Biographies of the other authors are not available. 